\title{
Psychometric Validation of the Maslach Burnout Inventory (MBI) Adapted to Moroccan Teachers
}

\author{
Abdeslam Amri' ${ }^{1}$, Zakaria Abidli ${ }^{2}$, Mounir Bouzaaboul ${ }^{1}$, \\ Ziri Rabea $^{1}$, Ahmed Omar Touhami Ahami ${ }^{1}$, Abdelmajid Soulaymani ${ }^{2}$ \\ ${ }^{1}$ Laboratory of Cognitive Behavioral Neuroscience and Applied Nutrition, Faculty of Science. Faculty of Sciences, \\ Ibn Tofail University, Kenitra, Morocco, ${ }^{2}$ Laboratory of Genetics and Biometrics, Faculty of Sciences, Ibn Tofail \\ University, Kenitra, Morocco
}

\begin{abstract}
Introduction: The literature on burnout research shows that more than $90 \%$ have used the Maslach Burnout Inventory (MBI).

Objective: The aim is to establish the psychometric properties in terms of reliability, sensitivity and validity of the Maslach Burnout Inventory (MBI) and to adapt it to the context of Moroccan teachers.

Method: This work was carried out through a process of translation and reverse translation of the original instrument by adapting the elements to the teaching profession. To determine the psychometric characteristics, we used Cronbach's alpha to determine reliability, exploratory factor analysis to extract factors and determine dimensionality, and confirmatory factor analysis to test the validity of the statistical model. Validation of the instrument was carried out on a sample of 170 teachers working in public schools in Ouezzane region (Morocco).

Results: Items 4, 5, 7, 9, 12, and 22 were relatively incoherent with respect to the other items, they were eliminated. The factorial analysis made appear three factors whose are explain $58.22 \%$ of the total variance. Cronbach's $\alpha$ for the whole questionnaire (0.761), for emotional exhaustion (0.819), depersonalization (0.850) and sense of personal accomplishment (0.785). The confirmatory factor analysis (CFA) showed satisfactory adjustment indices $(\mathrm{X} 2=164,938-\mathrm{df}=101-\mathrm{CFI}=.937-\mathrm{TLI}=.915-\mathrm{RMSEA}=.037-\mathrm{P}$ close $=.984)$.
\end{abstract}

Conclusion: We propose to use Maslach's Theoretical Model for the Moroccan Sample in Future Research on the burnout of Moroccan teachers.

Keywords: Psychometric, Validation, Maslach Burnout Inventory, Moroccan, Teachers.

\section{Introduction}

Burnout is a chronic response to emotional and interpersonal stressors in work that is exhausting,

\section{Corresponding Author:}

\section{Abdeslam Amri}

Laboratory of Cognitive Behavioral Neuroscience and Applied Nutrition. Faculty of Science. Faculty of Sciences, Ibn Tofail University, Kenitra, Morocco e-mail: abdeslam.amri@uit.ac.ma cynical and ineffective ${ }^{1}$. It manifests itself in three forms: emotional exhaustion, depersonalization and the loss of the sense of personal accomplishment ${ }^{2}$. These three components are represented in the Maslach Burnout Inventory (MBI), whose success of his versions increases $^{3}$. However, psychometric comparisons of MBI note both similarities and differences between several cultures $^{4-5}$ and cross-cultural comparisons of teacher burnout have yielded mixed results. For this reason, it is interesting to produce a diagnostic tool for professional burnout that is valid and specific to the cultural context of teachers in order to manage the risk that threatens the 
psychological health of these resources, which play a main role in the development of society ${ }^{6}$.

In Morocco, there is no tool for assessing burnout valid and adapted to the context of teachers. For this reason, the objective of this research is to establish the psychometric validity of an Arabic translation of MBI based on empirical research and to provide a validated translation of the MBI specific to Moroccan teachers.

\section{Material and Method}

\section{A. Measuring Instrument: Maslach Burnout} Inventory MBI Questionnaire: The MBI is composed of 22 questions that explore three scales: emotional exhaustion ( 9 items), depersonalization (5 items), and the sense of personal accomplishment $(8 \text { items })^{7}$.

1. Response modalities and quotation: The answers are given on a 7-point scale from "never" to "every day".

- Emotional Exhaustion (EE) is assessed by adding items: $1,2,3,6,8,13,14,16$ and 20 (result between 0 and 54),

- Depersonalization (DP) by adding items 5, 10, 11,15 and 22 (result between 0 and 30).

- Personal Accomplishment (PA) by adding the items: 4, 7, 9, 12, 17, 18, 19 and 21 (result between 0 and 48) ${ }^{7}$.

2. Translation of the questionnaire: The translation of the MBI was done by two bilingual individuals working in the field of education, in collaboration with colleagues and specialists in the field of education to make the questionnaire specific to teachers. A review was conducted following the commentanalysis after the administration of the questionnaire to a pilot group of 20 teachers working in the public education sector.

B. Sample: The validation of the instrument was carried out with a sample of 170 teachers working in public educational institutions belonging to the management of Ouezzane (Morocco). 37.6\% ( $\mathrm{n}=$ $64)$ female and $62.3 \%(n=106)$ male. The mean age is $38.3 \pm 8.9$ years.

C. Statistical analyses: The approach recommended by Anderson and Gerbing (Anderson J, 1988) ${ }^{8}$ to do a sequential analysis was adopted. The reliability of the instrument was examined by calculating the
Cronbach's $\alpha$ coefficient for the three dimensions of the inventory. Then the data were subjected to an Exploratory Factor Analysis (EFA) to extract the factors and test the dimensionality of the questionnaire. The factor structure was studied by performing a principal component analysis (PCA) and varimax rotation. The Kaiser - Meyer - Olkin (KMO) measurement was calculated to assess the adequacy of the sampling. Ideally, the KMO should be greater than 0.60 . The internal coherence of the global scale and the dimensions retained after rotation were evaluated using the Cronbach $\alpha$.

For the adequacy of the model, we used different adjustment indices: the index square root approximation error of Steiger Lind (RMSEA), the normalized adjustment index (NFI), the Tucker - Lewis index (TLI), the comparative adjustment index (CFI) and $\mathrm{P}$ close. These indices are interpreted according to critical thresholds ${ }^{9}$.

In general, values below 0.05 and 0.08 for the RMSEA are considered to indicate good and acceptable data adequacy respectively. The CFI and TLI should be greater than 0.9. The Pclose must be close to 1; the fit of the model is considered good when critical thresholds and listed standards are met. The statistical analyses were performed under SPSS 21 and AMOS 20.

\section{Results}

1. Internal consistency of theoretical dimensions and deletion of items: Using Nunnally's classification, which normally accepts Cronbach's $\alpha$ index above $0.70^{10}$, the removal of items 4 and 12 from the entire scale increased this index from 0.68 to 0.75 .

For depersonalization, the deletion of items 22 and 5 allowed Cronbach's $\alpha$ to increase from 0.67 to 0.76 . For the sense of personal accomplishment, the elimination of items 9 and 7 allowed Cronbach's $\alpha$ to increase from 0.63 to 0.77 . Items $4,5,7,7,9,12$, and 22 were therefore relatively incoherent with respect to the other items, so we decided to eliminate them from further analysis.

2. Exploratory Factor Analysis (EFA): The factorial analysis in principal axes with varimax rotation made appear three factors whose eigen values are greater than 1 and explain $58.22 \%$ of the total variance. The three dimensions are well defined and distinct on their respective factors; Thus, the first factor, which includes six items $(1,2,3,3,6,8,14)$ constituting the emotional exhaustion dimension, 
explains $37.05 \%$ of the total variance. the second, with six items $(10,11,13,13,15,16,20)$ constituting the depersonalization dimension explains $13.79 \%$ of the total variance and the third with four items $(17,18,19,21)$ constituting the personal achievement dimension, explains $7.37 \%$ of the total variance (Table 1).

Table 1: Matrix of components after rotation, percentages of explained variance and Cronbach's $\alpha$ of the three dimensions of the Maslach Burnout

Inventory specific to Moroccan Teachers.

\begin{tabular}{|c|c|c|c|}
\hline & \multicolumn{3}{|c|}{ Dimensions } \\
\hline & EE & DP & AP \\
\hline QI1 & ,72 & & \\
\hline QI2 & ,79 & & \\
\hline QI3 & ,57 & & \\
\hline QI6 & ,72 & & \\
\hline QI8 & ,65 & & \\
\hline QI14 & ,66 & & \\
\hline QI10 & & 71 & \\
\hline QI11 & & ,69 & \\
\hline QI13 & & ,71 & \\
\hline QI20 & & ,59 & \\
\hline QI15 & & 80 & \\
\hline QI16 & &, 55 & \\
\hline QI17 & & & ,75 \\
\hline QI18 & & & ,79 \\
\hline QI19 & & & ,73 \\
\hline QI21 & & & ,75 \\
\hline Cronbach's $\alpha$. & 0,81 & 0,85 & 0,79 \\
\hline$\%$ of the explained variance & 37,05 & 13,79 & 7,38 \\
\hline$\%$ of total variance explained & & 58,22 & \\
\hline $\begin{array}{l}\text { Precision measurement } \\
\text { of Kaiser-Meyer-Olkin } \\
\text { sampling (KMO) }\end{array}$ & & 0,88 & \\
\hline $\begin{array}{l}\text { Bartlett sphericity test: } \\
\text { Chi-square approximate } \\
\text { Dl } \\
\text { Significance of Bartlett }\end{array}$ & & $\begin{array}{l}1096,95 \\
120 \\
\mathbf{0 . 0 0 0}\end{array}$ & \\
\hline
\end{tabular}

EE: Emotional exhaustion, DP: Depersonalization, PA: Sense of personal accomplishment

In view of these results, the translation and application of the MBI in Arabic to a sample of Moroccan teachers keeps the dimensionality of the original construct. Cronbach's $\alpha$ on the global scale (0.761) as well as those for emotional exhaustion (0.819), depersonalization $(0.850)$ and sense of personal achievement $(0.785)$ are acceptable (Table 1).

3. Analysis of the structure of the dimensions and sensitivity of the Maslach Burnout Inventory specific to Moroccan Teachers: In order to clarify the existing relationships between the dimensions of the Maslach Burnout Inventory specific to Moroccan Teachers, the score for each dimension was calculated. Depersonalization (DP) and emotional exhaustion (EE) scores are positively correlated with each other, and negatively correlated with personal achievement (PA) scores, confirming the results of the MBI theoretical model. Similarly, to test the sensitivity gender and age variance analyses were conducted. The matrix of correlations between the three sub-dimensions and the age and gender variables shows the sensitivity of this 16-item questionnaire (Table 2)

Table 2: Means, standard deviations,correlations between the dimensions of the Maslach Burnout Inventory specific to Moroccan Teachers and between these dimensions and age and gender variables.

\begin{tabular}{|l|c|c|c|c|c|}
\hline Variables & Means & $\begin{array}{c}\text { Standard } \\
\text { deviation }\end{array}$ & EE & DP & AP \\
\hline EE & 21,73 & 9,18 & & & \\
\hline DP & 13,62 & 10,01 &, $61^{* *}$ & & \\
\hline AP & 15,05 & 6,51 &,$- 21^{* *}$ &,$- 445^{* *}$ & \\
\hline Age & 38,30 & 8,92 &, $16^{*}$ &, 04 &, 01 \\
\hline Gender & & &,$- 21^{* *}$ &,$- 28^{*}$ &, 05 \\
\hline
\end{tabular}

** The correlation is significant at level 0.01 (bilateral).* The correlation is significant at level 0.05 (bilateral).

4. Confirmatory Factor Analysis: Finally, by respecting the quality of the adequacy indices of the Schermelleh-Engel, Moosbrugger \& Muller model (Schermelleh-Engel K, 2003)9, the Confirmatory Factor Analysis (CFA) carried out on the data of our sample showed that our tested three-dimensional model presents satisfactory adjustment indices. X2 $=164.94 ; \mathrm{DF}=101 ; 0 \leq \mathrm{X} 2 \leq 2 \mathrm{df} ; \mathrm{RMSEA}=0.03$; CFI $=0.93$ above the critical threshold of 0.9 and the $\mathrm{P}$ close of 0.98 is very close to 1 (Table 3 ). 
Table 3: Quality thresholds of the model's adequacy indices According to Schermelleh, Engel, Moosbrugger \& Muller 9

\begin{tabular}{|l|c|c|c|}
\hline \multirow{2}{*}{ Indices } & \multirow{2}{*}{ Model } & \multicolumn{2}{|c|}{ Thresholds of acceptability } \\
& \multicolumn{2}{|c|}{$\begin{array}{c}\text { Good adequacy } \\
\text { Acceptable adequacy }\end{array}$} \\
\hline Xf & 164,93 & $\begin{array}{c}0 \leq \mathrm{X} 2 \leq 2 \mathrm{df} \\
0 \leq 164,938 \leq 202\end{array}$ & $2 \mathrm{df}<\mathrm{X} 2 \leq 3 \mathrm{df}$ \\
\hline CFI & 101 & & \multicolumn{2}{|c|}{$\mathrm{CFI} \geq 0,90$} \\
\hline RMSEA & 0,93 & $\begin{array}{c}0 \leq \mathrm{RMSEA} \leq 0,05 \\
0 \leq 0,037 \leq 0,05\end{array}$ & $0,05<\mathrm{RMSEA} \leq 0,08$ \\
\hline P close & 0,03 & $\begin{array}{c}0,10<\mathrm{p} \leq 1 \\
0,10<0,984 \leq 1\end{array}$ & $0,05 \leq \mathrm{p} \leq 0,10$ \\
\hline
\end{tabular}

In view of this table, our model shows a good adequacy with the observed data, which allows us to formulate the validation of the three-dimensional character of the MBI translated into Arabic specific to
Moroccan teachers. The 16-item Moroccan version specific to MBI teachers proposed by this research is therefore a validated structure.

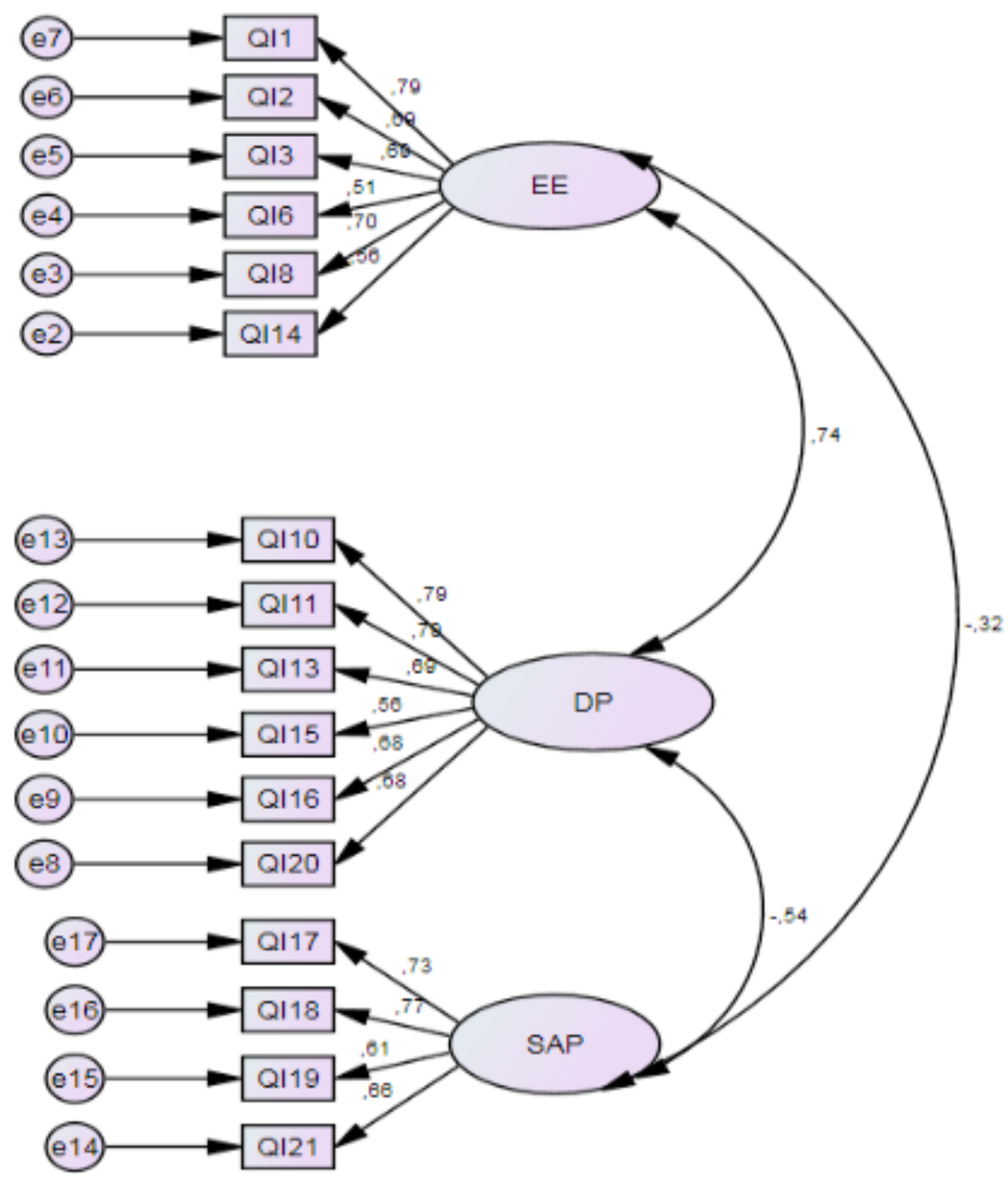

Figure 1: Confirmatory Factorial Analysis (CFA) performed by the maximum likelihood estimation method. MBI-ESM three-factor model $(16$ items $) .(X 2=164,938-\mathrm{df}=101-\mathrm{CFI}=.937-$ TLI $=.915-$ RMSEA $=.037-$ $P$ close $=.984$. 


\section{Discussion}

The objective of this study was to construct and test the factor structure, internal reliability, sensitivity and validity of a scale of measurement translated into Arabic of professional burnout for Moroccan teachers, designed according to Maslach's three-dimensional theoretical model. The 16-item tool was validated on a representative sample of teachers from the Ouezzane exercising in northern Morocco, using a sequential analysis (internal coherence, EFA and CFA). Several validation tests have shown the inconsistency of some theoretical items of the 22-item MBI with the characteristics of the samples studied, which forced the researchers to exclude certain items to improve their model. Ellenge Denton and al, in the United States of America and Jamaica, had excluded 10 items, the teacher-specific version was validated at 12 items ${ }^{11}$. Aluja and al, in Spain, had excluded five items; the teacher-specific version for them was validated at 17 point ${ }^{12}$. Concerning our study, we excluded the items 4 , $5,7,9,12$, and 22, and we kept a 16-item model. This inconsistency of these items could be explained by the characteristics of the teaching profession, the Moroccan language and culture. This confirms Wheeler's idea that further studies are needed on item-level analyses to explain the divergent results across occupations, languages and cultures ${ }^{13}$. The exploratory factorial analysis has retained the three dimensions for the 16item MBI-ESM, which is consistent with several studies like those of Byrne and al in Canada ${ }^{14}$, Yadama GN and Drake B in China ${ }^{15}$, and Boles JS and al in the United States ${ }^{16}$. This study reveals a deviation from items 13, 16 and 20, which are theoretically associated with emotional exhaustion in the initial version, towards depersonalization. This deviation of theoretical items from one dimension to another has been observed in Abu-Hilal's study in $2018^{17}$. Confirmatory Factor Analysis (CFA) confirmed the validity of the 16item MBI specific to Moroccan teachers with very satisfactory data adjustment indices (X $2=164.938$ - df $=101-\mathrm{CFI}=0,937-\mathrm{TLI}=0.915-\mathrm{RMSEA}=0.037$ and $\mathrm{P}$ close $=0.984$ ). This valid instrument is proposed for the evaluation of burnout among Moroccan teachers in future research. Concerning internal consistency, MBIES reliability analyses through studies systematically give Cronbach's alphas that vary between 0.8 and 0.9 for emotional exhaustion (EE), between 0.50 and 0.79 for depersonalization (DP), and between 0.69 and 0.82 for personal achievement (AP) ${ }^{12}$. For this study, Cronbach's alphas on the global scale (0.761) as well as those of emotional exhaustion (0.819), depersonalization (0.850) and sense of personal accomplishment (0.785) are satisfactory and comparable to those reported in several versions, indeed, the Cronbach alpha value of emotional exhaustion (0.819) is goobut remains lower than that of several studies. On the contrary, the depersonalization rate $(0.850)$ is higher (Table 4$)$.

Table 4: Cronbach's Alpha of the different dimensions of MBI-ES according to different studies. DPS: Depersonalization towards students. DPJ: Depersonalization towards work

\begin{tabular}{|c|c|c|c|c|c|}
\hline \multirow{2}{*}{ Studies } & \multirow{2}{*}{ Teaching cycle } & \multicolumn{4}{|c|}{ Cronbach's Alphas of the different dimensions of MBI-ES } \\
\hline & & $\mathbf{E E}$ & \multicolumn{2}{|c|}{ DP } & $\mathbf{A P}$ \\
\hline Maslach \& Jackson $(1986)^{7}$ & Primaryschool & 0,90 & \multicolumn{2}{|c|}{0,79} & 0,76 \\
\hline Fernando \& Perez $(1996)^{18}$ & Primary and secondary & 0,88 & \multicolumn{2}{|c|}{0,61} & 0,82 \\
\hline Won sunchen $\&$ al $(2014)^{19}$ & - & 0,91 & \multicolumn{2}{|c|}{0,78} & 0,78 \\
\hline Abu hilal \& al $(2018)^{17}$ & Primary and secondary & 0,87 & $\begin{array}{l}\text { DPS } \\
0,47\end{array}$ & $\begin{array}{l}\text { DPJ } \\
0,79\end{array}$ & 0,76 \\
\hline Our study (2019) & Primary and secondary & 0,81 & \multicolumn{2}{|c|}{0,85} & 0,78 \\
\hline
\end{tabular}

\section{Conclusion}

The results of the factors analysis of our study corroborate of Maslach's theoretical model, and a 16-item MBI model with better adjustment indices, demonstrating high conceptual validity, was proposed for use in the research of the psychological assessment of Moroccan teachers' burnout.
Conflict of Interest: The authors declare that there are no conflicts of interest.

Ethical Approval: The procedures were carried out in accordance with the recommendations of the internal Ethics Committee of the Ibn Tofail University Kenitra. This procedure were examined and approved by the Committee. 
Source of Funding: This work is not financial.

\section{Reference}

1. Maslach C, Schaufeli WB, Leiter MP. Job Burnout. Annu Rev Psychol. 2001;52(1):397422.

2. Maslach, C., \& Jackson, S. E. Burnout in organizational settings. Applied Social Psychology Annual, 1984: 5, 133-153.

3. Antoniou A-S, Ploumpi A, Ntalla M. Occupational Stress and Professional Burnout in Teachers of Primary and Secondary Education: The Role of Coping Strategies. Psychology. 28 mars 2013;04:349.

4. Abu-Hilal MM, el-Emadi AA. Factor structure and reliability of burnout among Emirati and Palestinian teachers. Psychol Rep. oct 2000;87(2):597610.

5. Richardsen AM, Martinussen M. Factorial validity and consistency of the MBI-GS across occupational groups in Norway. Int J Stress Manag. 2005;12(3):28997.

6. FannyMollard and Morgana Vecchia . Bachelor's thesis in pre-school and primary education. PDF [Internet]. [cited May 26, 2019]. Available on: https://docplayer.fr/116230681-Bachelorprofessional-membrance-in-school-primary-andprimary-fanny-mollard-and-organic-vecchia.html

7. Maslach, C., \& Jackson, S. E. Burnout in organizational settings. Applied Social Psychology Annual, 1984: 5, 133-153.

8. Anderson J, Gerbing DW. Structural Equation Modeling in Practice: A Review and Recommended Two-Step Approach. Psychol Bull. 1 janv 1988;103(3):41123.

9. Schermelleh-Engel K, Moosbrugger H, Müller H. Evaluating the Fit of Structural Equation Models: Tests of Significance and Descriptive Goodness-ofFit Measures. Method Psychol Res. 2003;8(2):2374.

10. Nunnally JC (1978). Théoriepsychométrique, 2nd Edn. New York, NY: McGraw-Hill.

11. Denton E, Chaplin WF, Wall M. Teacher Burnout: A Comparison of Two Cultures Using Confirmatory
Factor and Item Response Models. Int J Quant Res Educ. 2013;1(2):14766.

12. Aluja A, Blanch A, García LF. Dimensionality of the Maslach Burnout Inventory in School Teachers: A Study of Several Proposals. Eur J Psychol Assess. 2005;21(1):6776.

13. Wheeler DL, Vassar M, Worley JA, Barnes LLB. A Reliability Generalization Meta-Analysis of Coefficient Alpha for the Maslach Burnout Inventory. EducPsychol Meas. 1 févr 2011; 71(1):23144.

14. Byrne BM. The Maslach Burnout Inventory: Testing for factorial validity and invariance across elementary, intermediate and secondary teachers. J Occup Organ Psychol. 1 sept 1993;66(3):197212.

15. Yadama GN, Drake B. Confirmatory factor analysis of the Maslach Burnout Inventory. Soc Work Res. sept 1995; 19(3):18492.

16] :Boles JS, Dean DH, Ricks JM, Short JC, Wang G. The Dimensionality of the Maslach Burnout Inventory across Small Business Owners and Educators. J VocatBehav. 1 janv 2000;56(1):1234.

17. Maher Abu-Hilal, Said Al dafri, Hashem Kilani, Ali Kazem, Ibrahim Qaryouti, \& Hussein AlKharusi. Structure of Burnout among Omani Male and Female Teachers: Invariance of Structure across Gender. Journal of Educational and Psychological Studies - Sultan Qaboos University. 2018. 12; 739749

18. Fernando, J ., \& Perez, J. Un instrumento para medir que mazon professional en los Docentes : Adaptation Catalania del Maslach Burnout Inventry (MBI) an instrument to evaluate professional burnout in teachers: Catalonian adaptation of the MBI .Rivista de Ppsiquiatria de la facultad de medecine de la universidad de Barcelona. 1996.23;11-18.

19. Won Sun Chen .Translation, Cross-cultural Adaptation and Validation of the Malay Version of the Maslach Burnout Inventory (MBI) in Malaysia; International Journal of Social Science Studies. 2014;2:2324-8041. 